\title{
Webquest para el aprendizaje del contenido de derivadas por definición en la asignatura Cálculo a nivel universitario
}

Webquest for learning the content of derivatives by definition in the subject Calculus at university level

Osmel Vitriago Casadiego
osmelvitriago35@gmail.com
Código ORCID: 0000-0003-4312-353X
Unidad Educativa Divino Niño,
Venezuela

Venezuela

\author{
Victor David Badillo Mieres \\ badillovictor_10@hotmail.com \\ Código ORCID: 0000-0002-9274-2700
}

Unidad Educativa Ramón Pierluissi

Ramírez, Venezuela

\section{Artículo recibido en febrero 2020 | Arbitrado en marzo 2020 | Publicado en mayo 2020}

\section{Resumen}

La presente investigación tuvo como objetivo proponer una Webquest para el aprendizaje del contenido de derivadas por definición en la asignatura Cálculo I del tercer semestre de la mención matemática de la Facultad de Ciencias de la Educación-Universidad de Carabobo (FaCEUC). Esta se sustentó bajo las teorías de Ausubel (1983), el cual habla acerca del aprendizaje significativo, además de Cabero (2007) al realizar una integración de la tecnología en el ámbito educativo. El diseño fue de campo, no experimental, tipo proyectivo. Se tomó en cuenta una población conformada por nueve (9) estudiantes, donde se les aplicó un cuestionario de selección simple de veintidós (22) preguntas, como técnica de instrumento de recolección de datos. El estudio es un aporte didáctico tecnológico, que busca mejorar el proceso de enseñanza y aprendizaje en los estudiantes de la asignatura Cálculo I de la mención de Matemática de la FaCE-UC, Venezuela.

\footnotetext{
Abstract

The purpose of this research is to propose a Webquest for learning the content of derivatives by definition in the subject Calculus I of the third semester of the mathematical mention of the Faculty of Education Sciences - University of Carabobo (FaCE-UC). This is supported under the theories of Ausubel (1983), which talks about meaningful learning, in addition to Cabero (2007) when carrying out an integration of technology in the educational field. Field design, not experimental, projective type. A population made up of nine (9) students was taken into account, where a simple selection questionnaire of twenty two (22) questions was applied to them, as a technique of data collection instrument. The study is a technological didactic contribution, which seeks to improve the teaching and learning process in the students of the subject Calculus I of the mention of Mathematics of the FaCE-UC, Venezuela.
}

Palabras clave:

Webquest; aprendizaje; derivada
Keywords:

Webquest; learning; derivative 


\section{INTRODUCCIÓN}

En la actualidad la tecnología ha ocupado un lugar muy importante en la sociedad, tanto así que cada día revolucionan al mundo por completo. Además, han influido de manera significativa en la globalización y en la conexión de las distintas sociedades que integran el planeta, esto ha traído consigo el avance continuo de cada país que, a su vez, busca cada día mejorar el desarrollo integral de este. Cabe mencionar, que estas se encuentran estructuradas de acuerdo a un estudio específico para así brindarle a la sociedad todas las herramientas necesarias en cuanto al mejoramiento y perfeccionamiento de esta. Por esta razón, las tecnologías de la información y comunicación (TIC) forman parte de la estructura mencionada anteriormente, y a su vez se encuentran involucradas en la vida cotidiana de la sociedad, ya que están inmersas en las actividades que los seres humanos realizan diariamente.

Adicionalmente, las TIC han ido evolucionando de manera progresiva, pues cada día le brindan a la comunidad nuevas y diversas herramientas que sirven de ayuda para solventarle los distintos problemas que agobian al ser humano, esto trae consigo el avance continuo de la humanidad donde se puede visualizar a corto tiempo un futuro próspero e innovador lleno de diversos beneficios en pro del bienestar de la sociedad. Además, Rama y Chan (2017) afirman:

El futuro se nos ha transformado en un campo de investigación interdisciplinario creciente, y que mira lo real, el actual presente cambiante en tanto es en el aquí y ahora donde irrumpen algunas de las génesis de las próximas realidades. Antes, el porvenir estaba en el campo de la incertidumbre de las religiones. Luego, hemos visto paradigmas por los cuales el futuro está en el campo de los hombres, de la política y las acciones encaminadas a su construcción consciente. Hoy, sin embargo, con la expansión enorme de la ciencia y la tecnología en los últimos tiempos, no solo el futuro se nos ha tornado más complejo, sino que ha vuelto a irrumpir una reflexión sobre los posibles futuros asociados a una nueva dinámica de la ciencia y la tecnología relativamente inconmensurable. (p.l1)

En este orden de ideas, el mundo busca una nueva transformación, dando a conocer las nuevas realidades y el crecimiento que ha tenido la tecnología a nivel global, teniendo en consideración todos los campos de estudio existentes para así poder fomentar nuevas innovaciones que tengan como finalidad implementar nuevos ámbitos de estudio para el desarrollo de la ciencia y la tecnología. Es por ello que las TIC buscan innovar las herramientas existentes que lleve como objetivo asegurar un continuo conocimiento para lo largo de la vida.

Por consiguiente, el mundo ha exigido un nuevo desarrollo Educacional en donde las TIC juegan un papel protagónico e importante para el desempeño del estudiantado. Es decir que estas ayudan a que el estudiante genere un mejor interés en las clases, pues esto va a conllevar a que se produzca en el individuo un aprendizaje eficaz, eficiente y favorable. En tal sentido, la Organización de las Naciones Unidas para la Educación, Ciencia y Cultura (Unesco, 2008), señala que:

Gracias a la utilización continua y eficaz de las TIC en procesos educativos, los estudiantes tienen la oportunidad de adquirir 
capacidades importantes en el uso de estas. El docente es la persona que desempeña el papel más importante en la tarea de ayudar a los estudiantes en adquirir estas capacidades. Además, es el responsable de diseñar tanto las oportunidades de aprendizaje como entorno propicio en el aula que facilite el uso de las TIC por parte de los estudiantes para aprender y comunicar. Por esto, es fundamental que todos los docentes estén preparados para ofrecer esas oportunidades a sus estudiantes. (p. 02)

Por otro lado, las tecnologías de la información y comunicación han tenido un gran impulso para conquistar el ámbito escolar, tanto así que se ha tenido la necesidad de formar espacios que faciliten el proceso de aprendizaje requerido por el acelerado avance de las tecnologías. En este sentido, es importante que las instituciones educativas integren las tecnologías, ya que estas forman parte esencial de la sociedad y además las instituciones deben proveer el financiamiento y el buen uso de estos recursos. Cabero (2007) afirma:

Las nuevas tecnologías aportan a las cuestiones relacionadas con la enseñanza suficiente números de posibilidades y de tal grado de significación que obligan a buscar nuevos caminos didácticos acorde con las nuevas posibilidades, pero también obligan a una reflexión previa sobre su oportunidad y pertinencia. (p.22)

No obstante, las TIC le aportan a la educación unas gamas de nuevas estrategias para que el discente adquiera de manera directa un aprendizaje significativo que le permita una evolución continua de sus procesos cognitivos. De la misma forma estas, ayudan a buscar nuevos cambios que sirvan como herramientas pedagógicas que tengan como objeto principal la innovación e implementación de didácticas basadas para el crecimiento del aprendizaje de los estudiantes en el aula de clases.

En efecto, la educación venezolana en algunas ocasiones no pone en práctica las diversas herramientas innovadoras que aportan las tecnologías de la información y comunicación, esto trae como consecuencia que los docentes sigan implementando el mismo mecanismo tradicional que han venido acarreando durante muchos años, trayendo como resultado que los dicentes no adquieran nuevas metodologías de aprendizaje de los diversos contenidos que se le imparten durante toda su etapa de estudio.

Por lo tanto, Kaufman (2005) afirma que el trabajo de los docentes tiene que consistir en "planificar, diseñar e implementar un sistema eficiente y eficaz de aprendizaje, que responda a las necesidades de los estudiantes" (p. 55). En tal sentido, existen asignaturas muy complejas que requieren la implementación de las diversas herramientas que aportan las TIC para así mejorar y reforzar el aprendizaje de los contenidos que se les imparten a los estudiantes.

Por consiguiente, la asignatura de matemática no escapa de lo que se ha venido planteando anteriormente, ya que la mayoría de las veces los estudiantes ven dicha asignatura como obstáculo, esto se debe, a que el sistema que se ha venido implementando en los últimos tiempos no atrae todo el potencial intelectual que pueden demostrar los estudiantes en el aula de clase. Por tal motivo, los diferentes niveles educativos han venido exigiendo 
nuevas implementaciones innovadoras donde se ponga en práctica los distintos instrumentos que aportan las TIC, esto con la finalidad de originar varios mecanismos que sirvan para dar impulso a las distintas ejecuciones de materiales didácticos y tecnológicos que funcionen para mejorar y desarrollar los procesos de aprendizaje de los estudiantes en dicha asignatura mencionada anteriormente.

$\mathrm{Al}$ referirse a innovación según lo expresado por De la Torre (2005), es hablar de formación de actitudes, destrezas y hábitos, manejar estrategias, prever y superar resistencias, conocer procesos, afrontar conflictos, crear climas constructivos entre otros. Todo lo anteriormente descrito mejora la calidad de enseñanza, para optimizar el proceso educativo en una asignatura.

En relación a lo antes expuesto, el nivel de educación superior engloba un sin fin de contenidos esenciales, donde se observa la universalidad de la matemática que trae consigo un desarrollo esencial para así formar los procesos cognitivos que tengan que ver con los razonamientos lógicos, abstractos, espaciales y verbales. Cuando se examina esta cuestión surgen muchas confusiones acerca del sentido en que se utiliza la palabra "matemática". Por ello, la Universidad de Carabobo específicamente en la Facultad de Ciencias de la Educación de la mención matemática existe un gran déficit en el uso de las TIC para el aprendizaje del contenido de derivadas por definición dirigido a los estudiantes de la asignatura Cálculo I del tercer semestre, la cual ocasiona que los estudiantes no usen los recursos tecnológicos necesarios para un aprendizaje significativo y eficaz en dicho contenido.

Por ende, se propone la implementación de una Webquest para el aprendizaje del contenido de derivada por definición, para lo cual traerá consigo innovadoras aplicaciones en el aula de clases y que dispondrá una nueva didáctica aplicable para el proceso de enseñanza y aprendizaje de los discentes. En este sentido, los docentes tienen el deber de velar por el desarrollo de "potencialidades (habilidades, destrezas y actitudes) en sus estudiantes, encaminándolos hacia la apropiación de los recursos tecnológicos, lo cual se reflejará a medida que los estudiante usen el conocimiento científico y tecnológico en beneficio del progreso humano y social" (Ministerio del Poder Popular para la Educación, 2008: 4), lo que permitirá contribuir con la consolidación de un sistema educativo cónsono con los principios contemplados en el artículo 3o de la Constitución de la República Bolivariana de Venezuela (CRBV, 1999).

En este orden de ideas, surgieron los siguientes objetivos de investigación: 1.Diagnosticar los conocimientos previos de los estudiantes del contenido de derivadas por definición en la asignatura de cálculo I del tercer semestre de la mención matemática de la FaCE-UC; 2. Determinar la factibilidad de la propuesta; y 3. Diseñar una Webquest para el aprendizaje del contenido de derivadas por definición, para llegar a proponer una Webquest para el aprendizaje del contenido de derivadas por definición.

Es de hacer notar la importancia del estudio, al mencionar que la educación es un proceso desarrollador, vital para cualquier sociedad, ya que sirve como un pilar fundamental para el progreso continuo de una nación. Cabe destacar que cada pueblo cuenta con una educación basada y estructurada en sus creencias, culturas e ideales. Por consiguiente, ella también viene dada a través de los distintos cambios que han surgido de manera continua en la 
civilización, donde dichas transformaciones traen consigo una gama de variedades de programas que lo conforman. Una de estas, sin duda, son las TIC, ya que juegan un papel esencial e importante para el impulso continuo que exige cada día en el ámbito educativo. Cabe mencionar que estas se encargan de aportar diversas herramientas capaces de llevar consigo un proceso de enseñanza y aprendizaje mucho más eficiente.

Por dicha razón, la realización de la siguiente investigación buscó traer consigo la implementación de una Webquest que se encarga de aportar posibles soluciones a corto plazo a la grave situación que se ha venido presentando en la facultad. Cabe mencionar, una gran parte de dicha situación viene acarreada por motivos de la problemática subsistente transcurrida en los últimos años en Venezuela. Por tal motivo, esto ha repercutido de manera directa en el desarrollo y bienestar de la comunidad ucista, conllevado a que se agudice el índice de faltas de asistencia de los estudiantes en las aulas de clase, lo cual trae a su vez que ocurra un aumento continuo de la interrupción que se lleva a cabo en el proceso de enseñanza y aprendizaje.

Al mismo tiempo, no se puede pasar por desapercibido el importante papel protagónico que ejerce el educador en el ámbito educativo, ya que este a su vez también se ve perjudicado por la lamentable situación mencionada anteriormente, pues sin duda el docente tiene que buscar las diversas herramientas pedagógicas que traigan consigo la implementación de diferentes técnicas basadas principalmente en la innovación y producción de pensamientos, donde estos tienen que ir adaptados a las experiencias y necesidades sociales que le exige el entorno, esto con el fin de poder brindarle a los educandos un aprendizaje eficaz e importante para el progreso continuo de esta. Sin duda, cabe decir que lograr este cambio intelectual requiere de una aceptación, en repensar sobre la praxis educativa y reconstruir espacios conceptuales que hagan de la educación una transformación liberadora, implicando una transgresión ética a la que están sometidas las sociedades actuales.

\section{Estudios previos}

Con respecto al tratamiento, análisis y estudio de la aplicación de las Tecnologías de la Información y la Comunicación (TIC) en el ambiente educativo bajo la perspectiva constructivista, como elemento para el desarrollo del proceso de aprendizaje tiene una serie de antecedentes de importancia, entre los cuales se puede mencionar la investigación realizada por Meléndez (2014) titulada "La Webquest como un recurso de motivación para el aprendizaje de los temas de ciencias en estudiantes del 5to grado de secundaria de un colegio del cercado de Lima".

El estudio demuestra la gran importancia que posee la Webquest para el proceso de enseñanza y aprendizaje sobre los temas de ciencias, tecnología y ambiente. Por consiguiente, se evidenciaron aspectos positivos y negativos de la forma de enseñanza del docente. El docente ahora toma el rol de facilitador debido a la gran aplicabilidad de la Webquest. Adicionalmente, a raíz de la implementación de esta, se observa un incremento significativo del rendimiento académico en el segundo bimestre, trayendo a su vez un comportamiento beneficioso en las tres capacidades anteriormente mencionadas.

Desde hace mucho tiempo la sociedad ha sufrido cambios radicales que traen consigo buscarle diversas soluciones a las necesidades que se le presentan a diario. La tecnología ha influido de manera precisa en dichos cambios, por supuesto, cabe resaltar que estos cambios no se dan de la noche a la mañana, sino que 
forman parte de una evolución histórica. En este sentido, Toffler (citado por Unesco, 2008) señala que la creatividad y la receptividad es importante para enfrentar el futuro. Además, es oportuno destacar que no es obligatorio que una teoría sea cierta para que sea de gran utilidad.

Las TIC aportan una gran variedad de herramientas que ayudan al avance progresivo de la sociedad. Además, favorecen diversos cambios positivos que traen consigo un desarrollo continuo para el bien común de esta. La Unesco (2008) plantea una economía global que se traslada a países en vías de desarrollo, donde estos enfrentan problemas económicos, sociales, políticos, educativos, tecnológicos, entre otros. Estos buscan soluciones precisas para resolver dicha problemática. Al mismo tiempo, la nueva sociedad de la información o del conocimiento cuya visión del mundo es más multicentrica y multicultural, ofrece a dichos países una gama de oportunidades que sirven como impulso para el desarrollo sostenible de convenios entre países desarrollados y no desarrollados.

Por otra parte, Ausubel (1983) plantea que el aprendizaje del estudiante depende de la estructura cognitiva previa a los conocimientos o información que el educando maneje. Entiéndase por estructura cognitiva al conjunto de ideas que un individuo posee en un determinado campo de conocimiento, así como su organización. El autor, en su texto Psicología Educativa: Un punto de vista cognoscitivo, expresa lo siguiente:

Un aprendizaje es significativo cuando los contenidos: son relacionados de modo no arbitrario y sustancial (no al pie de la letra) con lo que el alumno ya sabe. Por relación sustancial y no arbitraria se debe entender que las ideas se relacionan con algún aspecto existente específicamente relevante de la estructura cognoscitiva del alumno, como una imagen, un símbolo ya significativo, un concepto o una proposición. (1983, p. 18)

El aprendizaje significativo ocurre cuando una nueva información se conecta con la información preexistente en la estructura cognitiva, esto implica que se pueden aprender conceptos, ideas o proposiciones que funcionen como punto de anclaje. Por ejemplo, si damos los conceptos básicos de física como el concepto de sistema, temperatura, presión, conservación de energía, estos ya subyacen en la estructura cognitiva del educando y estos servirán de subsensores para nuevos conocimientos relativos a la termodinámica, tales como máquinas térmicas, turbinas de vapor, rectores de fusión, entre otros.

En consecuencia, la presente investigación propone una estrategia que aliente a los estudiantes a aprender y desarrollar, por medio de la teoría del aprendizaje significativo de Ausubel, una herramienta tecnológica con un material organizado que presente los contenidos caracterizados por ser claros y estables, relevante e inclusivo del contenido que se va a enseñar, en pro de establecer un puente entre los conocimientos previos y lo que el estudiante necesita conocer, antes de aprender nuevos contenidos. En este sentido, la disposición para el aprendizaje significativo debe relacionar de manera sustantiva y no literal el nuevo conocimiento con su distribución cognitiva. Además, que el material aprendido por el discente sea significativo y establezca la estabilidad necesaria que este debe poseer para constituir una conexión o puente de lo aprendido en clase. 
La integración escolar de las nuevas tecnologías se encuentra fundamentada en las TIC, las cuales juegan un papel muy importante para el desarrollo del proceso de enseñanza-aprendizaje en un discente. Además, estas aportan muchas variedades de herramientas que pueden traer consigo un repertorio de conocimientos, actitudes y aptitudes que se le brindan al receptor con la finalidad de que este obtenga un aprendizaje mucho más eficiente y significativo con referencia a un contenido.

Las nuevas tecnologías aportan a las cuestiones relacionadas con la enseñanza suficientes números de probabilidades y de tal grado de significación que obligan a buscar nuevos caminos didácticos acordes con las nuevas posibilidades, pero también obliga a una reflexión previa sobre su oportunidad y pertinencia. (Cabero, 2007, p. 22)

En consecuencia, los instrumentos que brindan las tecnologías han venido desarrollando un proceso innovador para el ámbito educativo, esto con la finalidad de poner en práctica un sinfín de diferentes posibilidades que se adapten a las nuevas exigencias que requiere la sociedad. Lo anterior tiene por objeto traer consigo la apertura de diferentes caminos didácticos que funcionen para poder brindarle a los estudiantes diferentes oportunidades para mejorar su enseñanza.

Por otra parte, Quintana e Higueras (2009) establecen que la Webquest es un mecanismo muy útil que brinda las TIC, con la finalidad de ofrecerle a los estudiantes una herramienta de trabajo mucho más eficiente y que a su vez traiga consigo desarrollar el proceso de enseñanza y aprendizaje del discente en un tema determinado.
Cabero (2007) instituye que la Webquest se encuentra estructurada bajo cinco apartados los cuales son: 1) Introducción: según Quintana y Higueras (2009) definen que esta se encarga de "explicar los objetivos y los contenidos de la webquest" (p.7). 2) Tarea: según Cabero (2007) establece que "este apartado se proporciona al alumno una clara descripción de lo que tendrá que hacer al finalizar la webquest" (p. 225). 3) Proceso: según Quintana e Higueras (2009) expone que el proceso es una "propuesta organizacional y planificada de la tarea o trabajo en la cual se indican los recursos y documentos de consulta en línea" (p.ll). 4) Evaluación: según Cabero (2007) establece que en este apartado se indica cómo evaluar el trabajo realizado y para ello se detallan los criterios de evaluación en los cuales se basa la evaluación" (p. 228) y 5) Conclusión: según Quintana e Higueras (2009) definen este apartado como el "resumen de lo que se ha hecho y aprendido" (p. 1l).

Conjuntamente, esta herramienta cuenta también con dos apartados extra de manera complementaria que facilitan su identificación. Estos son: la portada, al inicio de la Webquest y guía didáctica al final. En resumidas cuentas, quizás lo más interesante de esta herramienta tecnológica es que el profesorado puede asumir, sin grandes costos, y con provecho del factor tiempo, la generación de material de enseñanza destinado a sus estudiantes usando la información y servicios útiles en la web. Además, de manera muy simple, los docentes pueden crear una Webquest tecnológicamente, mientras tenga la posibilidad de crear un documento con hipervínculos, puede crear una webquest, esto significa que puede ser creada por medio de conocimientos básicos que no impliquen 
poseer saberes avanzados en el área de programación.

\section{MÉTODO}

Con la finalidad de responder las incógnitas señaladas en el planteamiento del problema y que han servido de guía para la formulación de los objetivos, se indica la forma de cómo responder a esas inquietudes, para ello se procede, en primer lugar, a definir el tipo y diseño de la investigación, los sujetos de la investigación y procedimiento de la investigación. En segundo lugar, se indican las técnicas e instrumentos de recolección de datos, validez y confiabilidad del instrumento.

En este apartado se detalla el tipo de investigación, el cual Pallela y Martins (2012) lo definen como "la clase de estudio a realizar en la cual este se orienta sobre la finalidad general de estudio" (p. 88). Asimismo, esta investigación se desarrolló bajo un alcance de tipo proyecto factible, ya que el estudio está determinado a dar fin a una necesidad específica, por tal motivo se propone una Webquest para el aprendizaje de derivadas por definición en la asignatura de Cálculo I del tercer semestre de la mención de Matemática de la FaCE-UC, con la intención de que los estudiantes aprendan de manera clara y asertiva el contenido a tratar.

El presente estudio se realizó bajo un diseño de campo no experimental, ya que se observaron acciones que suceden en un entorno de la vida cotidiana para luego analizarlos. Además, la Universidad Pedagógica Experimental Libertador (UPEL, 2016) define la investigación de campo como el análisis sistemático de problemas en la realidad, con el propósito bien sea de descubrirlos, interpretarlos, entender su naturaleza y factores constituyentes, de ahí surge la aplicación de un único instrumento para recaudar la información.

En el caso de la presente investigación, la población o sujetos de estudio estuvo conformada por nueve (9) estudiantes de la asignatura de Cálculo I de la mención de Matemática de la Facultad de Ciencias de la Educación de la Universidad de Carabobo del turno de la mañana (sección ll) y del turno de la noche (sección 90).

Por otro lado, según Hernández, Fernández y Baptista (2014), la muestra "es un subgrupo de la población de interés sobre el cual se recolectarán datos, y que tiene que definirse y delimitarse de antemano con precisión, además de que debe ser representativo de la población" (p. 173). En este sentido, dicha muestra, dado que la característica de la población es pequeña y finita, se tomará como unidades de estudio a seis (6) sujetos de los que integran dicha población.

Es necesario resaltar los procedimientos realizados en este estudio, para ello se describen los siguientes pasos: a) Diagnóstico de los conocimientos previos, momento donde se aplicó un instrumento, el cual midió todo lo relacionado a los conocimientos precedentes que son requeridos para la derivada por definición; b) Factibilidad de la propuesta: En este se enfatizó la factibilidad de la propuesta y su aplicabilidad por ser fehaciente, puesto que la Facultad de Ciencias de la Educación posee los recursos necesarios para la misma; y c) El diseño de la propuesta presenta la estructura acorde para lograr el mejor cumplimiento en la ejecución del proceso de enseñanza y aprendizaje.

Cuando se refiere a la validez del instrumento, esta debe ser la ausencia de sesgos, la cual puede establecerse a través de 
distintos métodos como validez de contenido, de criterio, de constructo, validez externa e interna.

Por otra parte, Pallela y Martins (2012) afirman que la confiabilidad "es definida como la ausencia de error aleatorio en un instrumento de recolección de datos" (p. 164). El método de confiabilidad usado en la presente investigación fue la Correlación de Pearson o Confiablidad de Reaplicación de Prueba que según Ruiz (2002) esta "consiste en administrar dos veces una misma prueba a un mismo grupo de sujeto en un intervalo relativamente corto de tiempo (no más de tres meses entre una y otra medición)" (p.59).

El instrumento de recolección de datos que se aplicó para obtener la información de los estudiantes consistió en un cuestionario, previa aplicación de los criterios de validez y confiabilidad.

\section{RESULTADOS}

Estos datos fueron analizados, codificados y tabulados en términos absolutos y porcentuales, estableciendo con claridad la distribución de frecuencias de las respuestas dadas, además, de una representación gráfica que facilita la comprensión y entendimiento del análisis cuantitativo al momento de llevar a cabo la interpretación de los mismos.

El análisis correspondiente de la muestra total de estudiante en formación, con la intención de determinar si los discentes manejan la dimensión Algebra Elemental, la cual arrojó que el 56\% de los estudiantes manejan la dimensión anteriormente expuesta, donde el 13,9\% respondió incorrectamente y el 30,5\% no contestó y además, la dimensión con un total de 100\% de manejo por los estudiantes es la interpretación gráfica.
Por otra parte, el 45,8\% no contestó correctamente, a lo que indica la dimensión indeterminaciones. El 66,7\% maneja de manera concreta la extensión de trigonometría. En este sentido, la dimensión ecuación de la recta no dio ninguna conclusión porque no se obtuvo una información concreta de la misma, debido a que tanto para los estudiantes que contestaron correctamente, como los que contestaron poseen el mismo porcentaje, por ende no se da una conclusión muy clara acerca de esta dimensión y la dimensión teoría de limite se ubicó con el $45,8 \%$ del cual no contestaron correctamente, demostrando que la mayoría de los estudiantes no manejan la teoría de límite.

En resumidas cuentas, se puede verificar de manera propicia que el $53,8 \%$ de los estudiantes manejan de manera general las dimensiones que se evaluaron en la aplicación del instrumento y el 46,2\% de los estudiantes no poseen habilidades y destrezas en las dimensiones anteriormente mencionada.

Por otra parte, al realizar el estudio de factibilidad se pudo evidenciar, con respecto a la parte económica, en el proyecto factible diseñado. Así, el costo que generará diseñar una Webquest para el aprendizaje del contenido de derivadas por definición en la asignatura de Cálculo I del tercer semestre, es mínimo comparado con los múltiples beneficios que este aportará a la Facultad de Ciencias de la Educación de la Universidad de Carabobo, Venezuela. Además, no producirá gastos en hardware, recursos humanos, energía eléctrica ni servicio de Internet, pues la institución cuenta con todos estos recursos de manera gratuita.

Cabe destacar la ventaja económica que representa para los docentes como creadores de sus propios recursos y los estudiantes como usuarios finales en: hojas, lápices, 
marcadores, impresiones, entre otros. El único gasto generado es el servicio de Internet que utiliza el docente al planificar fuera de la institución.

En relación a la factibilidad técnica, se refiere a los recursos necesarios tales como herramientas, equipos, habilidades entre otros, para poder desarrollar de manera precisa cualquier producto, generalmente se alude a los elementos tangibles. "El proyecto debe considerar si los recursos técnicos actuales son suficientes o deben complementarse". (Brien, 2001, p. 27)

El aspecto técnico en el último objetivo de esta investigación relacionado al diseño de una Webquest para el aprendizaje de derivadas por definición de la asignatura de cálculo I del tercer semestre de la mención matemática de la FaCE-UC, se puede ejecutar ya que la institución cuenta con los recursos tecnológicos necesarios para tal fin.

Siguiendo el orden de las ideas anteriores fue necesario revisar la factibilidad operacional, la cual es referida a todos los recursos que intervienen en algún tipo de actividad, esta depende de los recursos humanos que participan durante el desarrollo del proyecto, la cual es expresada por Brien (2001) como: "Durante esta etapa se identifican todas aquellas actividades necesarias para lograr el objetivo, se evalúa y determina todo lo necesario para llevarla a cabo" (p.28).

Por otra parte, la factibilidad operativa de esta investigación fue netamente muy positiva, pues la Facultad de Ciencias de la Educación cuenta con el apoyo y disposición de un personal de primera categoría capacitado, dado que dicha facultad es pionera en el desarrollo investigativo de las Tecnologías de la información y comunicación.

\section{CONCLUSIONES}

La incorporación de las Tecnologías de la Información y la Comunicación (TIC) al contexto educativo es un proceso que involucra los conocimientos de las herramientas tecnológicas y sus potenciales pedagógicos. En este sentido, Internet se presenta como un recurso que forma parte de las TIC, permitiendo la creación de recursos de aprendizaje que facilitan los procesos de enseñanza.

Además, la praxis educativa ha exigido una mejora donde el profesional debe ser flexible, comprometido y a formarse constantemente para aportar recursos más sustanciosos a los discentes. Adicionalmente, las Tecnologías de la Información y Comunicación conllevan a aplicaciones infinitamente adaptables a cualquier plan educativo para la mejora del proceso de enseñanza y aprendizaje.

En este orden de ideas, se hace como propuesta el diseño de una Webquest para el aprendizaje del contenido de derivadas por definición, esto para innovar la praxis, debido a que es un recurso necesario para la base educativa de hoy en día, dado que esta facilita el aprendizaje en el aula y puede reforzar todos aquellos conocimientos que no están de un todo claro.

Así mismo, esta propuesta se basó en que actualmente los estudiantes tienen como fuente de investigación el Internet y otros medios, pero precisamente la web será la que contemple el papel principal debido a que esta será el centro de actividades cuando se trabaja una Webquest.

El diseño de la propuesta se justifica, pues las tradicionales metodologías de enseñanza y aprendizaje están hoy en día basadas en la elemental transmisión de información, ya que 
no responden a las demandas socioculturales de nuestro tiempo, ni se ajustan a los principios de construcción del conocimiento que caracterizan a la mayor parte de reformas educativas. Es por ello que se hace necesario la búsqueda de herramientas tecnológicas que tomen en consideración los principios de: creatividad, calidad, competencia y colaboración, que permitan avanzar hacia la nueva sociedad del nuevo siglo e impulsar el desarrollo del país; estos serán como los pilares que sustentan el cambio formativo de cada discente.

En este sentido, pocas dudas existen sobre la necesidad de formar a través de la innovación, caracterizada por la mejora colaborativa de la práctica docente, no tratándose de una mejora individual, sino compartida, no sólo como proceso de cambio externo, sino también cambio interno de las personas implicadas (docentes y estudiantes), para mejorar la calidad de la enseñanza y aprendizaje.

Adicionalmente, esta propuesta se presenta como una alternativa para los estudiantes de Cálculo I del tercer semestre de la mención Matemática de la Facultad de Ciencias de la Educación que no han tenido la oportunidad de hacer uso de herramientas tecnológicas como la Webquest, así como para cualquier docente que desee actualizar sus conocimientos y utilizar Internet en forma educativa empleando la Webquest.

Por esta razón la presente Webquest se justifica, tomando en consideración que su uso representa un puente abierto en el refuerzo del proceso de enseñanza y aprendizaje. Está en cada docente hacer que el conocimiento sea una vía dinámica, previa planificación que tengan como base el uso de las Tecnologías de la Información y Comunicación (TIC). El conocimiento de un nuevo tema no se puede encerrar en cuatro paredes, va más allá, es real y las Webquest son una actividad actual que debe ser considerada como un aporte educativo tecnológico de vanguardia.

Por otro lado, esta Webquest permite a docentes multiplicar las posibilidades en el ámbito educativo, pues supone una forma de utilizar didácticamente Internet, plantear tareas donde la mayoría de los recursos se encuentran en la red. Las actividades están centradas en usar la información extraída de Internet y las elaboradas por el mismo, para analizar, sintetizar comparar entre otros, más que en la búsqueda. De esta manera, los estudiantes saben dónde encontrar dicha información esto evita que se pierda navegando en la red sin un rumbo fijo. El docente al convertirse en autor de la Webquest previamente debe seleccionar los sitios web que servirán de recurso y fuente de información.

Finalmente, se pretende que el docente tenga una visión general sobre el diseño, creación y publicación de sus propias Webquest, para brindar a los estudiantes técnicas que le permitan el aprendizaje cooperativo y colaborativo, además de ofrecer la posibilidad que desarrollen roles que les permita descubrir vocaciones, habilidades o potencialidades, de esta manera pueda manifestar sus motivaciones intelectuales o sus inquietudes profesionales.

El objetivo general de la propuesta que se presenta es: Diseñar una Webquest para el aprendizaje del contenido de derivadas por definición. Para ello se deben ejecutar los siguientes objetivos específicos: 1) Recopilar la información referente al tema a tratar de la Webquest; 2) Configurar los diferentes aspectos multimedia (videos, textos, enlaces, entre otros) de la Webquest y 3) diseñar una Webquest para afianzar el conocimiento del tema a tratar en la misma. 
Es de hacer notar, la propuesta quedó estructurada de manera tal que se atiendan los conocimientos previos (álgebra elemental, funciones y límites de funciones) para atender aquellas deficiencias que posean los estudiantes en esta parte. Ahora bien, una vez cubierta la parte de los conocimientos previos se estableció el tema principal de la propuesta, la cual trata de la derivada por definición. Adicionalmente, para poder observar dicha propuesta se establecieron tres (3) módulos:

- Módulo I: Conocimientos previos.

- Módulo II: Derivada por definición.

- Módulo III: Ejercicios propuestos.

\section{REFERENCIAS}

Ausubel, D. P. (1983). Psicología Educativa: Un punto de vista cognoscitivo. México: Trillas

Brien, O. (2001). Estudios de Factibilidad. Madrid España: Editorial McGraw-Hill.

Cabero J. (2007). Nuevas tecnologías aplicadas a la educación. Madrid España: Editorial McGraw-Hill

Constitución de la República Bolivariana de Venezuela. Caracas. Gaceta Oficial $\mathrm{N}^{\circ}$ 5.453 del 24 de Marzo de 2000

De la Torre, S. (2005). Estrategias didácticas innovadoras. Barcelona, España: Ediciones Octaedro

Hernández, R., Fernández, C. y Baptista, P. (2014). Metodología de la Investigación. México: Editorial McGraw-Hill

Mendoza, J. (2015). Análisis de las Competencias de Tecnologías de Información y Comunicación
(TIC) de los Docentes en Formación de la mención matemática de la Facultad de Ciencias de la Educación de la Universidad de Carabobo. Caso: estudiantes de la asignatura módulo técnicas documentales. (Tesis de pregrado). Universidad de Carabobo, Valencia

Meléndez, M. (2014). La Webquest como recurso de motivación para el aprendizaje de los temas de ciencias en estudiantes del quinto grado de secundaria de un colegio del cercado de Lima. (Tesis de pregrado). Pontificia Universidad Católica del Perú, Lima. Recuperado:

http://tesis.pucp.edu.pe/repositorio/han dle $/ 123456789 / 5146$

Ministerio del Poder Popular para la Educación (2008).Manual de consulta, Modulo I: Las Tecnologías de la información y Comunicación versión l.1. Caracas, Venezuela. Recuperado de www.me.gob.ve

Rama, C. y Chan M. (2017). Futuro de los sistemas y ambientes educativos mediados por las TIC. México: Universidad de Guadalajara

Ruiz, C. (2002). Instrumento de Investigación Educativa. Barquisimeto, Venezuela: CIDEG

Unesco (2008). Estándares de Competencia en TIC para Docentes. Recuperado de http://portal.unesco.org/es/ev.php.URL_I $\mathrm{D}=41553 \&$ URL_DO=DO_TOPIC \& URL_SECTION=201.html

Universidad Pedagógica Experimental Libertador. (2016). Manual de Trabajos de Grado de Especialización y Maestría y Tesis Doctorales. Caracas, Venezuela: FEDUPEL 